\title{
Bloch modes and self-localized waveguides in nonlinear photonic crystals
}

\author{
Björn Maes, Peter Bienstman, and Roel Baets \\ Department of Information Technology, Ghent University-Interuniversity Micro-Electronics Center, \\ St.-Pietersnieuwstraat 41, 9000 Ghent, Belgium
}

Received February 11, 2004; revised manuscript received July 5, 2004; accepted October 10, 2004

\begin{abstract}
We present a modeling technique that uses eigenmode expansion to simulate infinite periodic structures with Kerr nonlinearity. Using a unit cell with Bloch boundary conditions, our iterative algorithm efficiently calculates self-consistent two-dimensional Bloch modes. We show how it can be used to study the band structure of nonlinear photonic crystals and to gain rapid insight in the operation of devices. Furthermore, we present nonlinear transversely localized guided modes, which are kinds of gap solitons or intrinsic localized modes, that induce their own waveguide through a photonic crystal without linear defects. (c) 2005 Optical Society of America

OCIS codes: 190.3270, 190.5530, 230.4320, 260.5950.
\end{abstract}

\section{INTRODUCTION}

Photonic crystals with nonlinear materials are subjects of active research. The Kerr effect, for example, makes it possible to dynamically tune the strong localization and dispersion effects in these metamaterials. Moreover, not only is it possible to adjust the linear properties but there are also new effects, such as localized nonlinear modes and gap solitons, to be studied. ${ }^{1-8}$

Along with theoretical and experimental progress, there is a need for efficient numerical tools with which to explore nonlinear devices and phenomena. We recently expanded the mode expansion technique for twodimensional Kerr nonlinear structures of finite size. ${ }^{9}$ Here we show how this algorithm can be adapted to model the self-consistent Bloch waves of infinite periodic structures. In this way we can observe the shifting of band structures with increasing amplitude. This ability is useful for device design, for which it is important to determine rapidly which frequencies and input intensities can couple to a guided Bloch mode.

As an application of our method we demonstrate the existence of self-localized waveguides in a two-dimensional photonic crystal without linear defects. These are Bloch modes with frequency in the bandgap, which are confined in the transverse direction because of the gap but propagate longitudinally as they carve their own guides. The self-localized waveguides can be seen as a variant of gap solitons ${ }^{7,8}$ because they overcome the longitudinal bandgap. But they can also be thought of as kinds of intrinsic localized modes, ${ }^{1,2}$ which are localized structures in discrete nonlinear lattices without defects, e.g. discrete solitons in waveguide arrays. ${ }^{5,6}$

We describe our modeling technique in Section 2. Applications of band structure calculations are shown in Section 3. In Section 4 we present self-localized waveguides and discuss several of their properties.

\section{MODELING TECHNIQUE}

In this section we give an overview of the simulation method. We begin by introducing the linear mode expansion technique, because this is the basis of the nonlinear calculations. Then we proceed with the algorithm to describe Kerr nonlinear materials. This algorithm can be used for both finite and periodic structures; therefore we point out the differences between them.

\section{A. Linear Mode Expansion}

In the linear mode expansion method one chooses a main propagation direction and divides the structure into sections that are invariant along this direction. An example of a simple structure with three parts is shown in Fig. 1. The forward-propagating field in such an invariant section can be described as a superposition of eigenmodes:

$$
\begin{aligned}
& \mathbf{E}(\mathbf{r})=\sum_{i} A_{i} \mathbf{E}_{i}\left(\mathbf{r}_{t}\right) \exp \left(-j \beta_{i} z\right), \\
& \mathbf{H}(\mathbf{r})=\sum_{i} A_{i} \mathbf{H}_{i}\left(\mathbf{r}_{t}\right) \exp \left(-j \beta_{i} z\right),
\end{aligned}
$$

where $\mathbf{r}_{t}$ is the transverse component of $\mathbf{r}$. Similar expressions can be derived for the backward field. Mode profiles $\mathbf{E}_{i}$ and $\mathbf{H}_{i}$ and their corresponding propagation constants $\beta_{i}$ are determined from the transversal index profile. Therefore, after calculation of the modes, the field in a section is reduced to a vector of complex mode amplitudes $A_{i}$.

To combine different sections we used the well-known mode-matching technique. This technique imposes continuity of the tangential total field components to infer transmission and reflection matrices for the mode amplitudes on the interface between two sections. At the end we combine these interface matrices with the propagation constants to derive a scattering matrix for the total struc- 


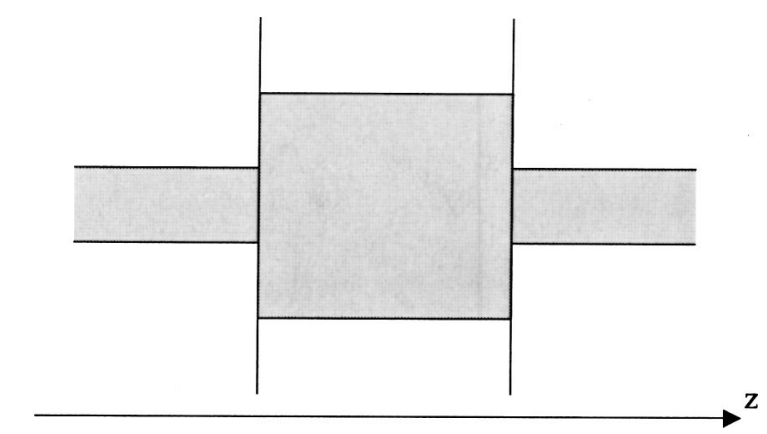

Fig. 1. Simple structure with three invariant sections along $z$.

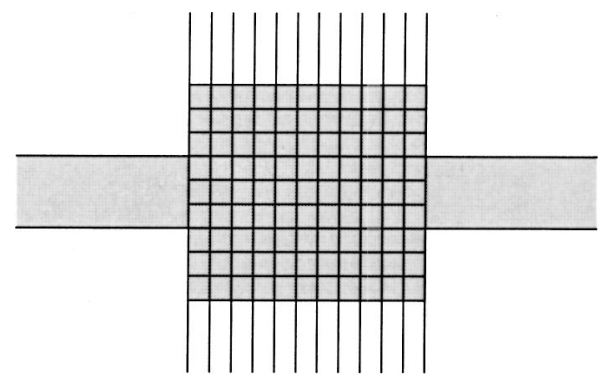

Fig. 2. Example of spatial discretization. The middle section is considered nonlinear.

ture. From this data we can obtain the fields throughout the entire structure by matrix manipulations. More information regarding the implementation of the linear mode expansion method can be found in Ref. 10.

\section{B. Nonlinear Extension}

To simulate instantaneous Kerr materials we use an iterative approach in combination with a spatial grid. Such nonlinearity means that the refractive index is dependent on the local field intensity $I=|\mathbf{E}|^{2}$ :

$$
n(\mathbf{r})=n_{0}+n_{2} I(\mathbf{r}),
$$

where $n_{0}$ is the linear index and $n_{2}$ is the Kerr coefficient. To model this index change we divide the nonlinear material into small rectangles. An example of such a grid is shown in Fig. 2. Each rectangle is assigned its index during each iteration. We can start by using the linear index, an estimate, or a previously calculated index distribution. By performing a linear eigenmode calculation as described above, we get the intensities at the center of each rectangle. ${ }^{11}$ This intensity distribution is used with Eq. (3) to update the index distribution. With the new index distribution we can calculate the fields again. This information provides us with another intensity distribution with which to update the indices, which completes another iteration cycle. If, after a number of iterations, the new index distribution is equal to the previous index distribution within a certain tolerance, we have converged to a solution of the full nonlinear problem.

The method is efficient because typically tens of eigenmodes are sufficient for accurate and rigorous modeling. In contrast, plane-wave techniques need hundreds or thousands of plane waves to simulate large index contrasts and field discontinuities. Finite-difference timedomain methods, however, have to calculate the entire evolution of a pulse-like excitation through the dis- cretized structure, leading to large time and memory requirements. We immediately get continuous-wave solutions, however, and only the nonlinear sections need to be discretized with a grid. Moreover, the modes and scattering matrices for linear subsections can be reused during each iteration because these data are indeed intensity independent. With reasonable parameters, convergence is reached in approximately ten iterations. Therefore all the simulations in this paper take seconds or minutes to complete on a modest workstation. We note that the method is rigorous, as there are no approximations to the Maxwell equations. Thus, in the limit of a fine grid and an infinite number of modes, the solutions are exact. A thorough description and study of the modeling method for finite structures are presented in Ref. 9.

\section{Finite and Periodic Structures}

Here we discuss the differences in applying the iterative method described for finite and periodic structures. For finite devices the input mode amplitude vector is constant during the calculations. We follow the evolution of the total scattering matrix, each time imposing the same input boundary condition.

For infinite periodic structures we describe the scattering behavior of a unit cell with reflection and transmission matrices in the following way:

$$
\begin{aligned}
& \mathbf{F}_{2}=\mathbf{T}_{12} \mathbf{F}_{1}+\mathbf{R}_{21} \mathbf{B}_{2}, \\
& \mathbf{B}_{1}=\mathbf{R}_{12} \mathbf{F}_{1}+\mathbf{T}_{21} \mathbf{B}_{2},
\end{aligned}
$$

where $\mathbf{F}$ and $\mathbf{B}$ denote mode amplitude vectors of forwardand backward-propagating fields, respectively. Subscripts 1 and 2 indicate left and right boundaries, respectively. Then we impose Bloch boundary conditions and rewrite Eqs. (4) as a generalized eigenvalue problem:

$$
\left[\begin{array}{cc}
\mathbf{T}_{12} & \mathbf{R}_{21} \\
0 & \mathbf{I}
\end{array}\right]\left[\begin{array}{c}
\mathbf{F}_{1} \\
q \mathbf{B}_{1}
\end{array}\right]=q\left[\begin{array}{cc}
\mathbf{I} & 0 \\
\mathbf{R}_{12} & \mathbf{T}_{21}
\end{array}\right]\left[\begin{array}{c}
\mathbf{F}_{1} \\
q \mathbf{B}_{1}
\end{array}\right],
$$

where $\mathbf{I}$ is the unity matrix, $q=\exp \left(i k_{B} p\right), k_{B}$ is the Bloch propagation constant, and $p$ is the period. ${ }^{12}$ From the set of resulting eigenvalues we select the mode of interest. This is normally the lowest-order value, as we are interested in lossless Bloch modes. The process of solving the eigenvalue problem as described above is performed at each iteration. However, because the unit cell is often small, the calculations are rapid. Simulating a finite structure with tens of periods, however, can be time consuming.

In contrast with the finite case, there is no input vector that we can keep constant during iteration. Another normalization parameter is needed to keep the calculations at each step self-consistent. To this end we use an excitation strength of the Bloch mode, which we define below. After fixing this strength at the start, we rescale the linear Bloch modes of the subsequent calculations.

There are different ways to define this excitation strength. We can use the flux of the Poynting vector through a boundary:

$$
P=\frac{1}{2} \int\left(\mathbf{E} \times \mathbf{H}^{*}\right) \cdot \mathrm{d} \mathbf{S},
$$


which we apply in Section 4 below for the waveguide modes, as $P$ immediately delivers a parameter of interest for waveguides. If one tries to calculate band-edge modes, the previous definition is not effective, because the flux approaches zero. Therefore, in these cases we keep the electrical energy in a period constant:

$$
U_{e}=\frac{\epsilon_{0}}{2} \int n^{2} I \mathrm{~d} V .
$$

A drawback here is the need to calculate an integral over the spatial period. We employ this normalization in Section 3 below.

In summary, by inspecting the guided Bloch waves we can derive a band structure that depends on the power flux or on the electrical energy of the corresponding mode. With the method just described it is thus possible for us to study efficiently in Section 3 below the tuning properties of devices.

\section{APPLICATIONS}

A. Nonlinear Photonic Crystal

Figure 3 shows results for a square lattice of square rods along the $\Gamma X$ direction. The simulations in this paper

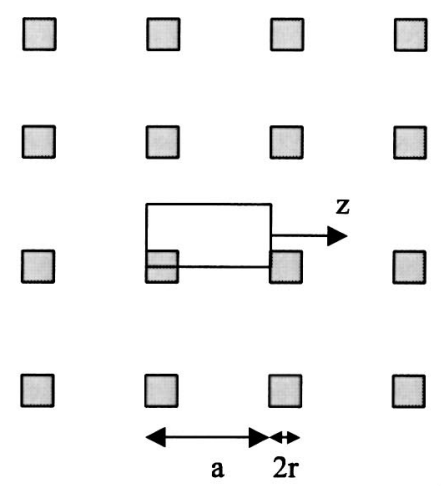

(a)

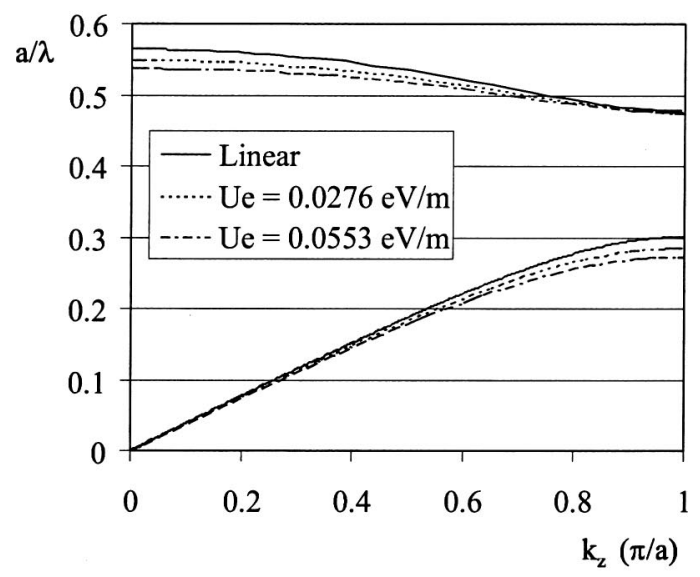

(b)

Fig. 3. Nonlinear photonic crystal. (a) Geometry: The rectangular box shows the unit cell that was used, exploiting symmetry. (b) Band structure: The solid curves show linear bands and dashed curves present nonlinearly adjusted bands.

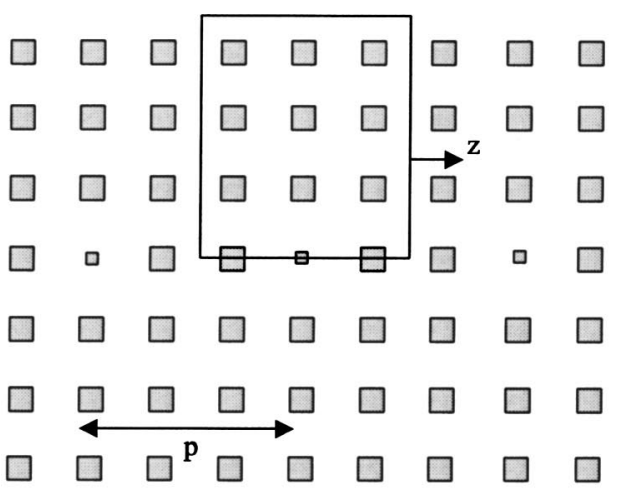

(a)

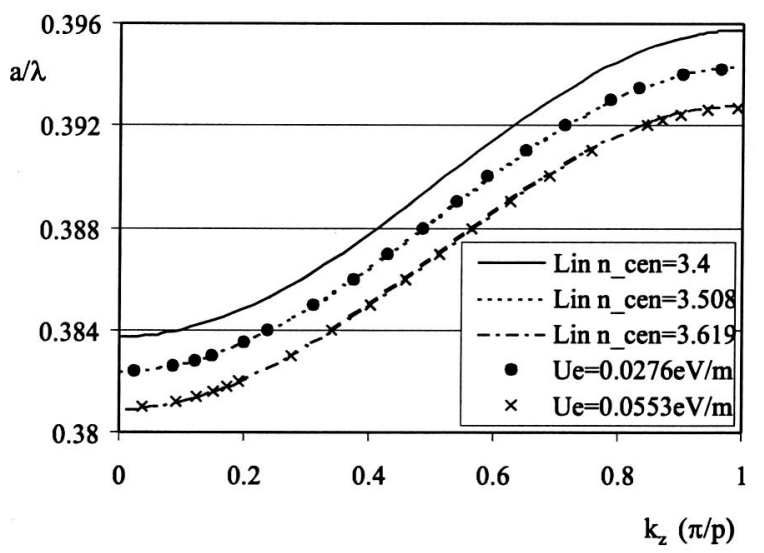

(b)

Fig. 4. Coupled-cavity waveguide. (a) Geometry: The rectangular box shows the unit cell used. Period $p=3 a$. (b) Band structure: curves, linear bands with defect rod index $\mathrm{n}_{-}$cen; filled circles and crosses, nonlinearly adjusted bands.

are for TM polarization, with the electric field parallel to the rods, but the method can also handle TE polarization. The rods have index 3.4 , and $n_{2}=1 \times 10^{-5} \mathrm{~m}^{2} / \mathrm{V}^{2}$ in an air background. The ratio of the sides of the rods to the crystal period is $2 r / a=0.25$. The linear photonic crystal has a TE gap at $a / \lambda=0.347-0.477$. Figure 3 shows the typical downshifting of the bands for positive $n_{2}$ with increasing energy $U_{e}$; see Eq. (7). Similar calculations have been performed. ${ }^{13-15}$ Applications of these band shifts are reported, e.g., in Ref. 15.

\section{B. Coupled-Cavity Waveguide}

In this subsection we calculate the dispersion relation of coupled-cavity waveguides in nonlinear photonic crystals. ${ }^{16}$ These waveguides are formed by evanescent coupling between neighboring resonator modes. In our case we form the monopole modes by lowering the diameter of a rod to $0.1 a$; see Fig. 4(a). The resonator spacing is $3 a$, and the same crystal lattice and polarization are used as previously. We consider that only the defect rod is nonlinear, with $n_{2}=1 \times 10^{-5} \mathrm{~m}^{2} / \mathrm{V}^{2}$. This is a good approximation, because the field in the resonator is strongest in the center.

The dispersion relationship for a linear coupled-cavity waveguide has a cosine form:

$$
\omega=\omega_{0}+\Delta \omega \cos \left(k_{z} p\right),
$$


where $p$ is the period; here $p=3 a .{ }^{16} \quad \omega_{0}$ and $\Delta \omega$ are determined by the resonance frequency of the mode and the nearest-neighbor overlap integrals. We retrieve this cosine form, as shown in Fig. 4(b). For the nonlinear bands there is again a downward shift. As a reference we also plot linear bands, determined with the mean nonlinear index of the central rod for a fixed $U_{e}$. Indeed, the nonlinear calculations should overlap these linear results.

\section{Limiter Device}

Many periodic nonlinear devices are based on shifting propagating modes in and out of the bandgap for various intensities. To gain insight into these devices, studying the infinite structure can rapidly provide information on geometry, operational frequencies or intensities, without the need for time-consuming simulations on a finite long structure. We illustrate this shortest procedure by modeling a recently proposed waveguide photonic crystal limiter. ${ }^{17}$ Its geometry and results are shown in Fig. 5. The same lattice as above is used but with a defect waveguide and larger nonlinear rods $\left(D=0.4 a, n_{2}=1 \times\right.$ $\times 10^{-5} \mathrm{~m}^{2} / \mathrm{V}^{2}$ ) alongside every two subperiods.

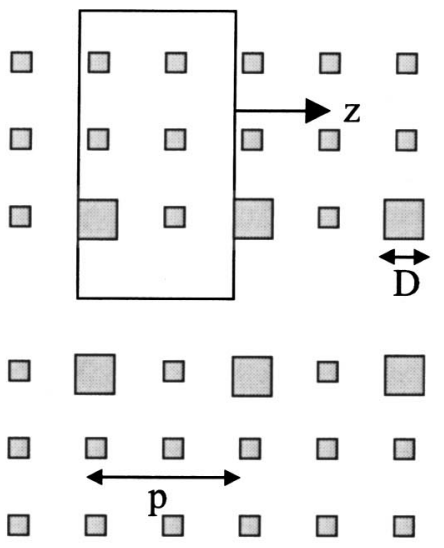

(a)

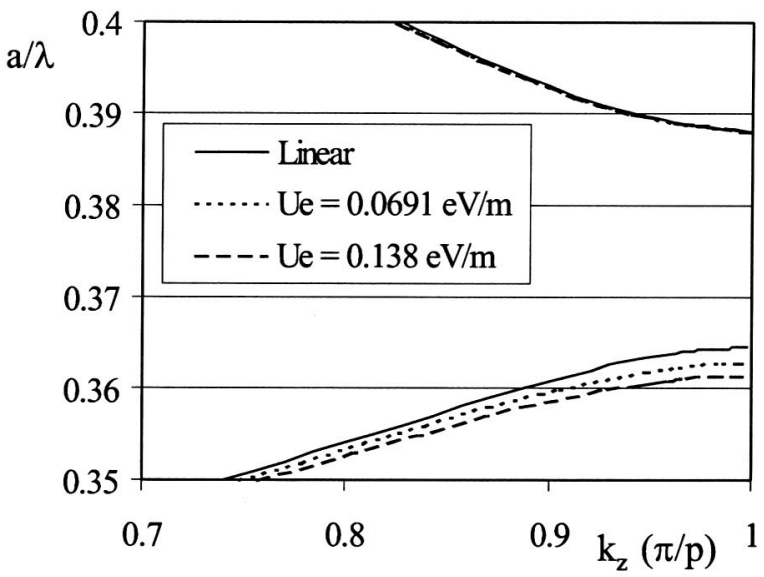

(b)

Fig. 5. Photonic crystal limiter. (a) Geometry: defect waveguide with larger nonlinear rods alongside. The rectangular box shows the unit cell used. (b) Waveguide bands: solid curves, linear bands; dashed curves, nonlinearly adjusted bands. The upper bands overlap. Period $p=2 a$.
The band structure shows two waveguide bands within the bandgap. Increasing the intensity shifts the lowest defect band downward. The upper defect band is quite insensitive to the nonlinearity. Field plots show indeed that the fields in this band are concentrated in the smaller linear rods along the waveguide, whereas in the lower band the fields are strongest in the large nonlinear rods. Thus, if we operate on top of the lowest waveguide band, low amplitudes will give a high transmission. But, as we increase the amplitude, our simulations indicate that the guided Bloch mode disappears below the working frequency, so we get limiting action.

\section{SELF-LOCALIZED WAVEGUIDES}

Waves with frequency within the bandgap are exponentially dampened in a linear photonic crystal without defects. However, with Kerr materials there are new effects to be studied, such as localized modes and gap solitons. Many of these nonlinear phenomena can be described from a linear point of view. For example, a spatial soliton in a bulk medium is the linear guided mode of the waveguide that it induces. ${ }^{18}$ From this idea we set out to find self-localized waveguides in a nonlinear photonic crystal, starting from a linear waveguide mode.

Figure 6 illustrates the waveguide modes. These are confined in one direction by bandgap effects but propagate in the other direction because the beam itself creates a defect waveguide. The same lattice as above is used but with a negative Kerr nonlinearity $n_{2}=-1 \mathrm{~m}^{2} / \mathrm{V}^{2}$. Note that we applied $\epsilon(\mathbf{r})=\epsilon_{0}+n_{2} I(\mathbf{r})$ instead of Eq. (3) for the calculations. These structures are a generalization of the localized modes presented in Ref. 4 and belong to the family of gap solitons. To our knowledge this is the first presentation of rigorous data on these self-localized waveguides. Modes confined in two directions have been theoretically studied. ${ }^{3,7,8}$ However, in the research presented in Ref. 7 a low-index contrast method was used to characterize moving or stationary gap solitons, whereas in Ref. 3 stationary modes in high-index contrast were considered. There is also a clear analogy with localized entities in waveguide arrays, such as Bragg solitons or discrete solitons, especially with the strongly localized discrete solitons that involve only a few coupled waveguides. $^{6}$ However, an important difference is that self-localized waveguides have to overcome a bandgap in the longitudinal direction.

To model these modes we use the technique of Section 2 but start with a seed. We lower the index of a center rod to create a linear defect waveguide. The linear calculation with this index gives an estimate of the needed flux and a starting index distribution. If we iterate from this distribution, with constant flux, and converge to a guided Bloch mode, we have reached a self-localized waveguide. After finding one mode we can change the frequency or the flux slightly to iterate to new modes quickly and map their properties.

In Fig. 6(a) the mode has one maximum on the rod in the center; therefore we call these modes on site modes. However we also found another type with two maxima of opposite sign on adjacent rods; these are intersite modes. A typical field profile of the intersite type is shown in Fig. 


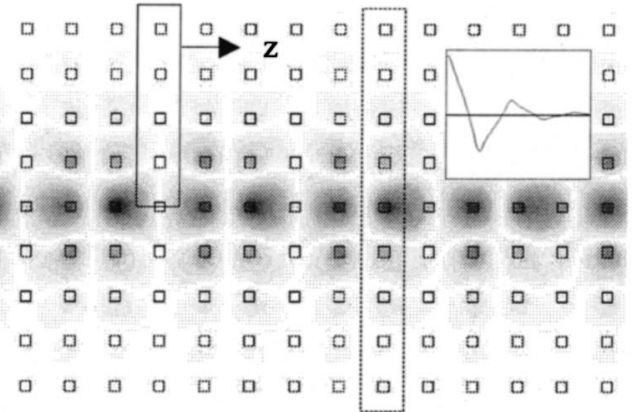

(a)

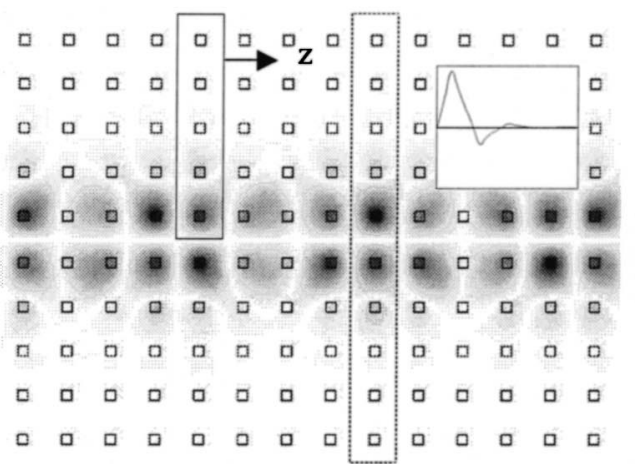

(b)

Fig. 6. Examples of the electric fields of two types of staggered self-localized waveguide. The smaller rectangular boxes show the unit cell that we used to find the mode. The larger rectangular (dashed) boxes are used for the study of stability. The mode is linearly propagated through a crystal with a refractiveindex distribution derived from the nonlinear calculation, which is limited to the small rectangular box. Insets, electric-field profiles in a transversal cut through the rod centers. (a) On-site mode with $a / \lambda=0.38$ and $P=1.2 \times 10^{-10} \mathrm{~W} / \mathrm{m}$. (b) Intersite mode with $a / \lambda=0.41$ and $P=9 \times 10^{-10} \mathrm{~W} / \mathrm{m}$.

6(b). These profiles are reminiscent of the types of localized mode reported in other situations. Thus the existence of similar modes in other propagation directions or geometries, e.g., airholes in material, is suggested. Moreover, in analogy with discrete solitons, modes with other topologies, e.g., flat-topped solitons, can be considered.

With our method it is possible to make the following arguments about the stability of self-localized waveguides. Because the method is iterative, it is possible to calculate only modes that are stable to small perturbations. However, the boundary condition along the lower edge of the unit cell (enclosed by unbroken rectangles in Fig. 6) determines the symmetry of the solutions and of the perturbations. From this we conclude that the on-site (intersite) modes are stable against symmetric (asymmetric) or even (odd) perturbations. We can test stability against other perturbations by applying Peierls-Nabarro potential ideas. ${ }^{2}$ According to this concept, intersite and onsite modes with the same topology can be seen as constituents of the same soliton. Assuming equal intensity, the component with the highest energy will be unstable and evolve into the stable component with the lowest en- ergy. This qualitative picture gives insight not only into stability but also into mobility and switching opportunities. If the soliton wants to move in the transversal direction, it has to change between the on-site and the intersite modes. The difference in energy determines the Peierls-Nabarro barrier that has to be overcome to change from one stable mode to the next via the unstable intermediate mode. Thus if the energies are the same the modes are expected to be mobile in the transverse direction. Note that there is always propagation in the longitudinal direction.

We have calculated the energy of both types of selflocalized waveguide for frequencies throughout the gap. Note that the two types have the same topology, that both are staggered, and thus that the previous PeierlsNabarro ideas can be applied. For each wavelength we simulated modes with $k_{z} a / \pi$ ranging from $\sim 0.4$ (large $P$ ) to 0.9 (small $P$ ), with the extra condition that the refractive indices remain larger than 1 . These seem the most stable modes numerically. Results are shown in Fig. 7. We can see that, near the lower-frequency band edge, the difference between the energies is negligible, which suggests mobile modes. As the frequency increases, the energy difference becomes larger. Assuming a constant power $P$, the on-site modes have the lowest energy, so they are stable, whereas the intersite component is unstable. We numerically confirmed the previous statement by performing calculations on the complete modes, using the dashed rectangles in Fig. 6. In this rectangular box we can slightly perturb the indices of a rod and start the iteration. This procedure injects the soliton with eigenmodes of the symmetry that are not present in the half-box simulations. For a frequency away from the lower band edge, even a small change of $0.1 \%$ in the indices of an intersite center rod is enough to push the iteration toward convergence on an on-site mode. The on-site modes, however, are extremely robust.

Next we study the transverse width of the modes by calculating the following simple deviation:

$$
\sigma=\left(\sum_{i} d_{i}{ }^{2} E_{i} \max / \sum_{j} E_{j}^{\max }\right)^{1 / 2},
$$

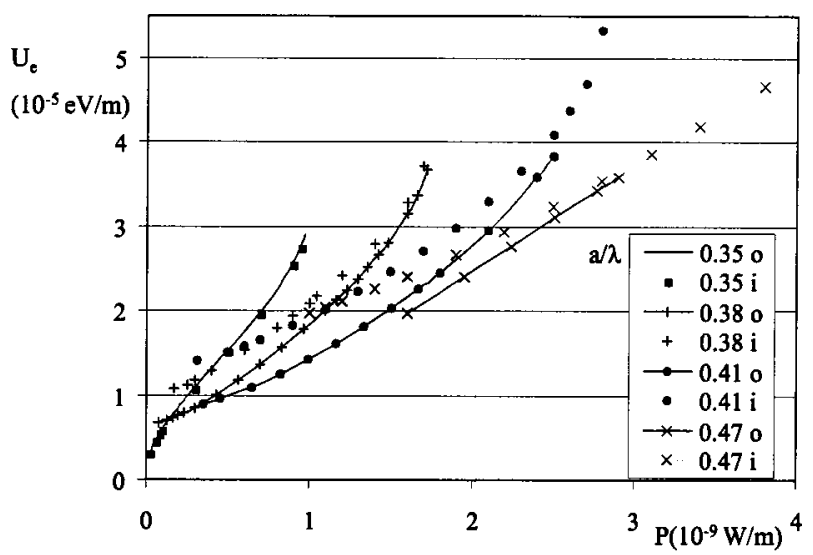

Fig. 7. Energy $U_{e}$ versus power $P$ for self-localized waveguides of different wavelengths. For values of $a / \lambda$, 0 and i mean on-site and intersite, respectively. 


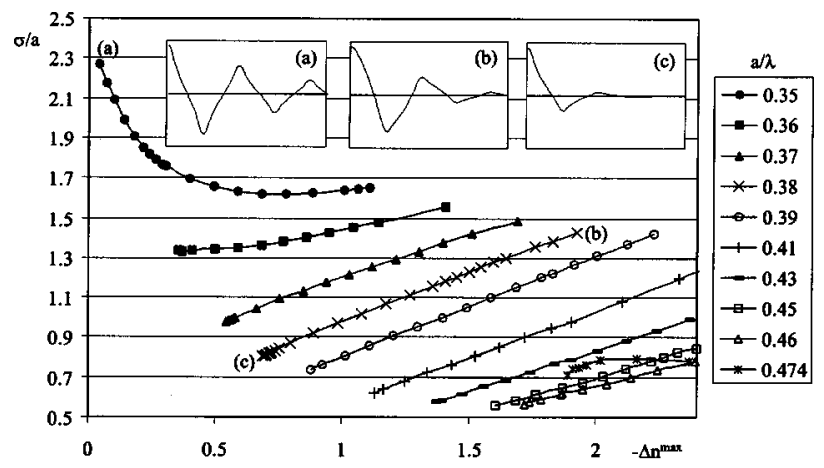

Fig. 8. Width $\sigma$ versus maximum refractive-index change $\Delta n_{\max }$ for on-site modes of various wavelengths $a / \lambda$. Insets, electricfield profiles for the three indicated points.

where $d_{i}$ is the distance from the center and $E_{i}{ }^{\max }$ is the maximum electric-field absolute value of $\operatorname{rod} i$. This deviation versus maximum refractive-index change for the on-site modes is shown in Fig. 8. For the intersite waveguides the curves are parallel. At the low-frequency gap edge we can see a strong broadening of the modes as we decrease the power. Although the nonlinear index change is lowest here, below 0.05 these modes lose the strong discrete localization and instead get a more continuous character. In the gap, decreasing the wavelength or the refractive-index change (or power $P$ ) leads to narrower waveguides. However, these trends no longer apply on the upper-frequency edge of the gap. We remark that the modes in the gap have a deviation smaller than 1.6 , so these are indeed strongly localized modes. If $\sigma$ is less than 1 , the waveguides will appear tightly bound on one center rod, as illustrated by the insets in Fig. 8 .

The broad modes at the low bandgap edge are analogous to the fundamental solitons in bulk nonlinear materials. ${ }^{19}$ However, there are important differences. First, the bandgap structure makes it possible to support waveguides with a low refractive-index core. Solitons in bulk material are guided in a self-induced high refractiveindex core by total internal reflection. Next, because of the anisotropic and discrete lattice the stability and dynamic behavior will be different from those in the continuous counterpart, especially for modes localized about a few periods. We have provided some insight into modal stability; however, a full analysis and a dynamic treatment are beyond the scope of the modeling method. We note that the minimum nonlinear index change for the solutions in the gap is theoretically high, near 0.3. However, it is clear that there are possibilities for optimization.

\section{CONCLUSIONS}

We have presented an efficient method with which to model band structures of two-dimensional devices with Kerr nonlinear materials. The influence of nonlinearity on guided Bloch waves has been shown in a perfect photonic crystal, in a coupled-cavity waveguide, and in a limiting device. Efficiency stems from rapid convergence to a solution and from the fact that only the nonlinear sections have to be discretized. Moreover, a few tens of eigenmodes are sufficient for accurate modeling of these wavelength-scale high-index contrast structures. With this technique we presented two types of self-localized Bloch mode, which are variants of gap solitons, and studied properties such as stability and width. The twodimensional photonic crystal and the simulation method can thus be used as a test case and a tool with which to analyze new kinds of intrinsic localized modes.

\section{ACKNOWLEDGMENTS}

B. Maes and P. Bienstman acknowledge a doctoral and postdoctoral fellowship, respectively, from the Flemish Fund for Scientific Research. Parts of this research were performed in the context of the Belgian Science Policy Interuniversity Attraction Pole project Photon.

B. Maes’s e-mail address is bjorn.maes@intec.ugent.be.

\section{REFERENCES}

1. A. J. Sievers and S. Takeno, "Intrinsic localized modes in anharmonic crystals," Phys. Rev. Lett. 61, 970-973 (1988).

2. D. Cai, A. R. Bishop, and N. Gronbech-Jensen, "Localized states in discrete nonlinear Schrödinger equations," Phys. Rev. Lett. 72, 591-595 (1994).

3. S. F. Mingaleev, Y. S. Kivshar, and R. A. Sammut, "Longrange interaction and nonlinear localized modes in photonic crystal waveguides," Phys. Rev. E 62, 5777-5782 (2000).

4. A. A. Sukhorukov and Y. S. Kivshar, "Nonlinear guided waves and spatial solitons in a periodic layered medium," J. Opt. Soc. Am. B 19, 772-781 (2002).

5. R. Morandotti, U. Peschel, J. S. Aitchison, H. S. Eisenberg, and Y. Silberberg, "Dynamics of discrete solitons in optical waveguide arrays," Phys. Rev. Lett. 83, 2726-2729 (1999).

6. F. Lederer, S. Darmanyan, and A. Kobyakov, "Discrete solitons," in Spatial Solitons, S. Trillo and W. Torruellas, eds. (Springer-Verlag, Berlin, 2001), pp. 269-292.

7. S. John and N. Aközbek, "Nonlinear optical solitary waves in a photonic band gap," Phys. Rev. Lett. 71, 1168-1171 (1993).

8. N. Aközbek and S. John, "Optical solitary waves in two- and three-dimensional nonlinear photonic band-gap structures," Phys. Rev. E 57, 2287-2319 (1998).

9. B. Maes, P. Bienstman, and R. Baets, "Modeling of Kerr nonlinear photonic components with mode expansion," Opt. Quantum Electron. 36, 15-24 (2004).

10. P. Bienstman and R. Baets, "Optical modelling of photonic crystals and VCSELs using eigenmode expansion and perfectly matched layers," Opt. Quantum Electron. 33, 327341 (2001).

11. CAMFR simulation software is freely available from http:// camfr.sourceforge.net/.

12. K. C. Huang, P. Bienstman, J. D. Joannopoulos, K. A. Nelson, and S. Fan, "Field expulsion and reconfiguration in polaritonic photonic crystals," Phys. Rev. Lett. 90, 196402 (2003).

13. P. Tran, "Photonic-band-structure calculation of material possessing Kerr nonlinearity," Phys. Rev. B 52, 1067310676 (1995)

14. V. Lousse and J. P. Vigneron, "Self-consistent photonic band structure of dielectric superlattices containing nonlinear optical materials," Phys. Rev. E 63, 027602 (2001).

15. A. Huttunen and P. Törmä, "Band structures for nonlinear photonic crystals," J. Appl. Phys. 91, 3988-3991 (2002).

16. A. Yariv, Y. Xu, R. K. Lee, and A. Scherer, "Coupledresonator optical waveguide: a proposal and analysis," Opt. Lett. 24, 711-713 (1999). 
17. T. Fujisawa and M. Koshiba, "Time-domain beam propagation method for nonlinear optical propagation analysis and its application to photonic crystal circuits," in Integrated Photonics Research, OSA Technical Digest Series (Optical Society of America, Washington, D.C., 2003), p. IME5.
18. A. W. Snyder, D. J. Mitchell, L. Poladian, and F. Ladouceur, "Self-induced optical fibers-spatial solitary waves," Opt. Lett. 16, 21-23 (1991).

19. R. Y. Chiao, E. Garmire, and C. H. Townes, "Self-trapping of optical beams," Phys. Rev. Lett. 13, 479-482 (1964). 